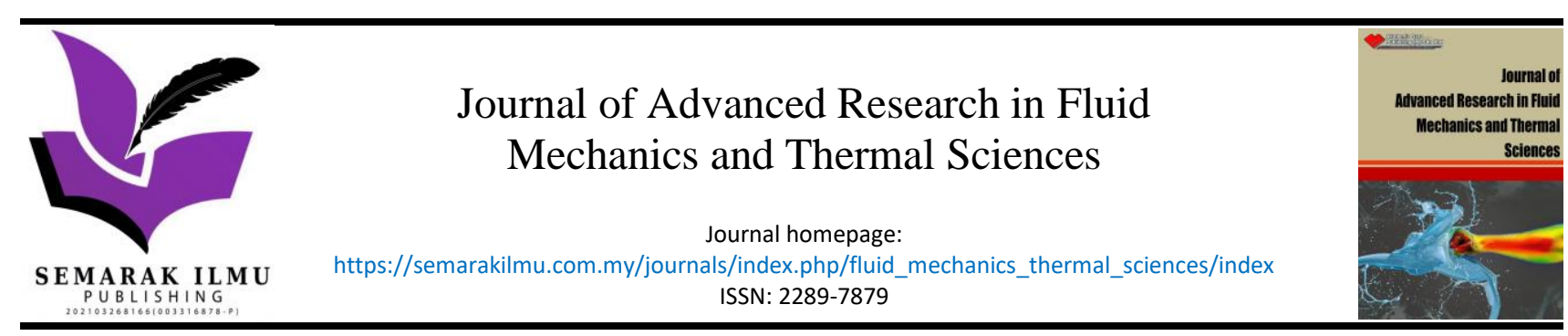

\title{
Investigation Of Dual-Pump Fiber Optical Parametric Amplifier Performance Driven by Energy Transfer Between Four-Wave Mixing Process
}

\author{
K. G. Tay ${ }^{1,}{ }^{*}$, Noran Azizan Cholan ${ }^{1}$, Nurulanati Othman ${ }^{2}$ \\ Faculty of Electrical and Electronics Engineering, Universiti Tun Hussein Onn Malaysia, 86400 Batu Pahat, Malaysia \\ 2 INTI International College Penang, Malaysia
}

\section{ARTICLE INFO}

\section{Article history:}

Received 8 June 2021

Received in revised form 31 October 2021

Accepted 7 November 2021

Available online 9 December 2021

\section{Keywords:}

2p-FOPA; gain; higher-order dispersion; fiber parameters; pump parameters; energy transfer; FWM

\section{ABSTRACT}

Fiber optical parametric amplifier (FOPA) is operated based on energy transfer from pump waves to signal wave and at the end of the fiber, an idler wave is generated. This process is called four-wave mixing (FWM). Even though effects of higher-order dispersion coefficients, fiber length, fiber nonlinearity, fiber attenuation, pump powers, pump wavelength separation $\Delta \lambda_{p}$ and distance of central pump wavelength with ZDW $\lambda_{c}-\lambda_{0}$ on gain profiles have been examined by previous researchers, but on different fiber or numerically studied using the Optisys system, analytical model or different amplitude equations. Thus, in this study, the above-mentioned parameters on the gain performance of dual pump fiber optical parametric amplifier (FOPA) using highly nonlinear shifted fiber (HNL-DSF) as a medium will be numerically investigated using ode45 function in Matlab. The gain at a certain wavelength can be obtained by solving 4 coupled amplitude equations with fiber loss and pump depletion that govern the four-wave mixing (FWM) process of pumps, signal and idler waves. Simulations results indicate positive $\beta_{2}$ gives poor or no gain, meanwhile, an addition of $\beta_{4}$ to negative $\beta_{2}$ widens the bandwidth, but there is no significant effect with the addition of $\beta_{6}$. Besides, an increase of fiber length, nonlinearity and pump powers improve gain performance, but an increase of fiber loss decays the gain amplitude. Increment of pump separation will enhance flatness of gain at wavelength far from central wavelength but results in an increase of gain reduction at the central wavelength. Lastly, $\lambda_{c}-\lambda_{0}$ must be positive, not too small and not bigger than $1.125 \mathrm{~nm}$ to get a high, broader and lesser ripples gain.

\section{Introduction}

The demand for ultra-fast telecommunication system has been dramatically increased due to the pandemic of COVID-19 which reduces face to face meeting and traveling but an increase of online meeting, teaching, presentation and training. Fiber-optical parametric amplifier (FOPA) which offers high flat gain and broad bandwidth of 10 to $100 \mathrm{~nm}[1,2]$ can increase the transport capacity of fiber

\footnotetext{
* Corresponding author.

E-mail address: tay@uthm.edu.my

https://doi.org/10.37934/arfmts.90.1.5263
} 
communication systems. Besides, FOPA also provides other functionalities such as adjustable center frequency and gain spectra, phase conjugation, wavelength conversion, pulsed operation for signal processing and 0-dB noise figure [3] has made FOPA overcome conventional amplifier such as RAMAN amplifier and (RA) and Erbium-doped fiber amplifier (EDFA). In a research conducted by Saris et al., [4] to enhance the gain of dual pump EDFA using OptiSystem software version 13, they have shown that dual pump EDFA has better gain performance at lower input signal power if compared to single pump EDFA.

Similarly, there are, $1 p$ and $2 p$-FOPAs. In practice, a good FOPA must exhibit high and flat gain as well as wide bandwidth. Even though $1 p$ - FOPA has a simple configuration but has poor flatness if compared to 2p-FOPA [5]. Contrary, 2p-FOPA can offer a higher, broader and flatter gain if compared to $1 p$-FOPA [6]. Hence, in this study, 2p- FOPA was considered. Energy cannot be created nor destroyed but can be transferred [7]. The process of energy transfer from dual pump waves with angular frequencies $\omega_{p 1}$ and $\omega_{p 2}$ to a signal wave with angular frequency $\omega_{s}$ and at the end an idler wave with angular frequency $\omega_{i}$ is generated is illustrated as shown in Figure 1. This process is called four wave mixing (FWM) such as $\omega_{p 1}+\omega_{p 2}=\omega_{s}+\omega_{i}$.

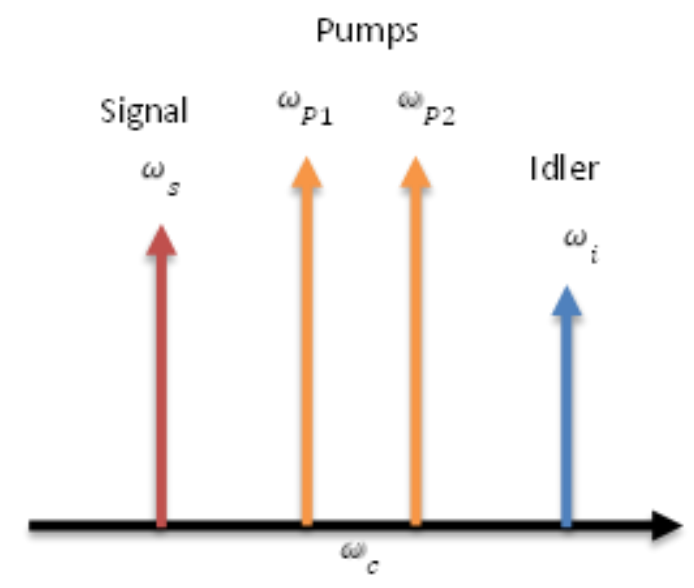

Fig. 1. Schematic diagram of FWM with two pump lights in the frequency domain

The performance of FOPA is influenced by fiber length, pump power, pump wavelength [8], fiber nonlinearity [9] and fiber attenuation [10]. For 2p-FOPA, pump separation $\left(\Delta \lambda_{p}\right)$ and center wavelength of two pump deviations from the fiber zero-dispersion wavelength $\left(\lambda_{c}-\lambda_{0}\right)$ do contribute to gain profile [9]. Boggio et al., [11] investigated experimentally gain characteristics of $2 p$ FOPA using conventional dispersion-shifted fiber. They obtained $37 \mathrm{~dB}$ gain with $\pm 1.5 \mathrm{~dB}$ ripple over $47 \mathrm{~nm}$ bandwidth.

Chen et al., [9] inspected effects of higher-order dispersion coefficients, $\beta_{4}$, fiber nonlinearity, fiber length, pump power, $\Delta \lambda_{p}, \lambda_{c}-\lambda_{0}$ towards the gain performance of $2 p$-FOPA using HNLF. They concluded that smaller and negative $\beta_{4}$ gives wider bandwidth, whereas longer fiber length improves gain but does not increase bandwidth. Meanwhile, an increment of fiber nonlinearity wider bandwidth, increase gain but reduce flatness., Similarly, an increase of pump power improves gain and bandwidth but reduce flatness. While large pump separation $(100 \mathrm{~nm})$ reduces the central gain to approximately zero. Contrary, when $\lambda_{c}-\lambda_{0}=0$, the gain bandwidth is broader, but when 
$\lambda_{c}-\lambda_{0}=1 \mathrm{~nm}$, the gain increases but its bandwidth decrease whereas if $\lambda_{c}-\lambda_{0}=-1 \mathrm{~nm}$, the bandwidth decease but its gain maintains.

Researchers [12] examined the performance of 2p FOPA using HNLF in terms of fiber nonlinearity, fiber length, higher-order dispersion up to order four using numerical simulation. They concluded that an increase of fiber nonlinearity improves the flatness and gain of $2 p$-FOPA, meanwhile an increase of fiber length and $\beta_{4}$ improves the gain. They obtained a net gain of $40 \mathrm{~dB}$ and $62 \mathrm{~dB}$ over a bandwidth $>100 \mathrm{~nm}$ on $400 \mathrm{~m}$ and $600 \mathrm{~m}$ fiber length respectively.

Performances of $1 p$ and $2 p$ FOPA using Optisys software were investigated in [13]. They obtained parametric amplification over a $50 \mathrm{~nm}$ gain bandwidth and $31 \mathrm{~dB}$ gain using $500 \mathrm{~m}$ highly nonlinear fiber (HNLF) for $1 p$ FOPA, whereas $26.5 \mathrm{~dB}$ gains over a $50 \mathrm{~nm}$ gain bandwidth for $2 p$ FOPA. Even though $2 p$ - FOPA gain is lower, but they give flat gain if compared to $1 p-F O P A$.

Similarly, the influence of fiber nonlinearity, fiber length, fiber attenuation, $\beta_{2}$ on the performance of $2 p$-FOPA was studied in [10] using an analytical model. They concluded that gain amplitude increased with the increase of fiber nonlinearity, fiber length and negative $\beta_{2}$ while gain decrease with an increase of fiber attenuation. Based on their simulation results, they used the optimized parameter and obtained $38 \mathrm{~dB}$ gain over a wide bandwidth of $228 \mathrm{~nm}$ using short length Photonic crystal Fibers (PCFs) with $\gamma>50$.

Othman [14] examined the effect of pump parameters on $2 p$ FOPA using HNL-DSF. The reported that high pump power increase gain and flatness of gain especially far from the central wavelength. Meanwhile, an increase of $\Delta \lambda_{p}$ flattening the gain far from the central wavelength but reduces gain at the center. Lastly, they suggested $\lambda_{c}-\lambda_{0}$ must be positive and small to get a wide and flat gain.

Even though the effects of several parameters that contribute to the gain characteristic of $2 p-$ FOPA have been studied either experimentally, or numerically, but on different fiber or numerically studied using the Optisys system, analytical model or different numerical model if compared to Mathematical Model in the next section. Thus, in this study, the effects of fiber length, fiber nonlinearity, fiber attenuation, higher-order dispersion coefficients up to $\beta_{6}$, pump powers, $\Delta \lambda_{p}$ and $\lambda_{c}-\lambda_{0}$ will be examined on the gain characteristic of $2 p$-FOPA using HNL-DSF.

\section{Mathematical model}

The non-degenerate FWM induced by 2 continuous-wave $(C W)$ pump $1\left(P_{1}\right)$ and pump $2\left(P_{2}\right)$ which transfer the energy to a signal wave $(s)$ and at the end an idler wave $(i)$ is generated is governed by conventional four-coupled amplitude equations as below:

$$
\begin{aligned}
& \frac{d A_{p 1}}{d z}=i \gamma\left[\left(\left|A_{p 1}\right|^{2}+2\left|A_{p 2}\right|^{2}+2\left|A_{s}\right|^{2}+2\left|A_{i}\right|^{2}\right) A_{p 1}\right]+2 i \gamma A_{p 2}^{*} A_{s} A_{i} e^{i \Delta \beta z}-\frac{\alpha}{2} A_{p 1} \\
& \frac{d A_{p 2}}{d z}=i \gamma\left[\left(\left|A_{p 2}\right|^{2}+2\left|A_{p 1}\right|^{2}+2\left|A_{s}\right|^{2}+2\left|A_{i}\right|^{2}\right) A_{p 2}\right]+2 i \gamma A_{p 1}^{*} A_{s} A_{i} e^{i \Delta \beta z}-\frac{\alpha}{2} A_{p 2} \\
& \frac{d A_{s}}{d z}=i \gamma\left[\left(\left|A_{s}\right|^{2}+2\left|A_{p 1}\right|^{2}+2\left|A_{p 2}\right|^{2}+2\left|A_{i}\right|^{2}\right) A_{s}\right]+2 i \gamma A_{i}^{*} A_{p 1} A_{p 2} e^{i \Delta \beta z}-\frac{\alpha}{2} A_{s}
\end{aligned}
$$




$$
\frac{d A_{i}}{d z}=i \gamma\left[\left(\left|A_{i}\right|^{2}+2\left|A_{p 1}\right|^{2}+2\left|A_{p 2}\right|^{2}+2\left|A_{s}\right|^{2}\right) A_{i}\right]+2 i \gamma A_{s}^{*} A_{p 1} A_{p 2} e^{i \Delta \beta z}-\frac{\alpha}{2} A_{i}
$$

where $z$ is propagation distance, $\gamma$ denotes the fiber nonlinearity, $\alpha$ represents the fiber loss, $\Delta \beta$ is linear phase - mismatch, $A_{j}$ represents amplitude for $\mathrm{j} \in\left\{p_{1}, p_{2}, s, i\right\}$ and $A_{j}{ }^{*}$ for $\mathrm{j} \in\left\{p_{1}, p_{2}, s, i\right\}$ indicates the complex conjugate of $A_{j}$. The linear phase-mismatch is given by [15] as

$$
\Delta \beta=2 \sum_{m=1}^{\infty} \frac{\beta_{2 m}}{(2 m !)}\left[\left(\Delta \omega_{s}\right)^{2 m}-\left(\Delta \omega_{p}\right)^{2 m}\right]
$$

where $\beta_{2 m}$, is the dispersion coefficient given by the $2 m^{\text {th }}$ derivative of mode-propagation constant $\beta(\omega)$, at the central frequency $\omega_{c}=\left(\omega_{p 1}+\omega_{p 2}\right) / 2=\left(\omega_{s}+\omega_{i}\right) / 2$. Meanwhile, $\Delta \omega_{s}=\omega_{s}-\omega_{c}$ and $\Delta \omega_{p}=\omega_{p 1}-\omega_{c}=\left(\omega_{p 1}-\omega_{p 2}\right) / 2$.

By taking $m$ up to 3 in expression (4), $\Delta \beta$ up to the sixth order dispersion coefficient $\beta_{6}$ is expressed as:

$$
\Delta \beta=\beta_{2}\left[\left(\Delta \omega_{s}\right)^{2}-\left(\Delta \omega_{p}\right)^{2}\right]+\frac{\beta_{4}}{12}\left[\left(\Delta \omega_{s}\right)^{4}-\left(\Delta \omega_{p}\right)^{4}\right]+\frac{\beta_{6}}{360}\left[\left(\Delta \omega_{s}\right)^{6}-\left(\Delta \omega_{p}\right)^{6}\right]
$$

For $m$ up to $3, \beta_{2}, \beta_{4}$ and are illustrated respectively by [16] as

$$
\beta_{2 m}=\sum_{n=2 m}^{\infty} \frac{\beta_{n 0}}{(n-2 m) !}\left(\omega_{p}-\omega_{0}\right)^{n-2 m}
$$

Where $\beta_{n 0}=\left.\frac{d^{n} \beta(\omega)}{d \omega^{n}}\right|_{\omega=\omega_{0}}$ is the dispersion coefficients calculated at the zero-dispersion frequency $\omega_{0}$.

By combining (5) and (6) for $m=1$ to 3 , the $\Delta \beta$ can be written as:

$$
\begin{aligned}
\Delta \beta= & \left.\left.\beta_{2}\left[\left(\Delta \omega_{s}\right)^{2}-\left(\Delta \omega_{p}\right)^{2}\right]+\frac{\beta_{4}}{3}\left[2\left(\omega_{c}-\omega_{o}\right)^{2}\right]\left(\Delta \omega_{s}\right)^{2}-\left(\Delta \omega_{p}\right)^{2}\right]+\frac{1}{4}\left(\Delta \omega_{s}\right)^{4}-\left(\Delta \omega_{p}\right)^{4}\right] \\
& \left.\left.+\frac{\beta_{6}}{24}\left[\left(\omega_{c}-\omega_{o}\right)^{4}\right]\left(\Delta \omega_{s}\right)^{2}-\left(\Delta \omega_{p}\right)^{2}\right]+\frac{1}{15}\left(\Delta \omega_{s}\right)^{6}-\left(\Delta \omega_{p}\right)^{6}\right]
\end{aligned}
$$

Here coefficients of $\beta_{2}, \beta_{4}$ and $\beta_{6}$ can be computed as given in [17].

Lastly, Eqs. (1)-(4) and (8) can be solved using fourth-order Runge-Kutta method [17-18], but in this study, ode45 function in Matlab was used due to its simplicity. Then, the parametric gains (in $\mathrm{dB})$ at the respective wavelength are calculated as:

$$
G=10 \log \frac{\left|A_{s, \text { out }}\right|^{2}}{\left|A_{s, \text { in }}\right|^{2}}
$$

Where $A_{s, o u t}$ and $A_{s, \text { in }}$ are output and input of amplitude signals using parameters in Table 1. 


\section{Table 1}

\begin{tabular}{lll} 
Parameters & & \\
\hline Parameter & Value & Unit \\
\hline Fiber length $(L)$ & 500 & $\mathrm{~m}$ \\
Fiber nonlinearity & 11.5 & $\mathrm{~W}^{-1} \mathrm{Km}^{-1}$ \\
Fiber loss $\alpha$ & 0.82 & $\mathrm{~dB} / \mathrm{km}^{-}$ \\
Pump1 wavelength $\left(\lambda_{p 1}\right)$ & 1552 & $\mathrm{~nm}$ \\
Pump2 wavelength $\left(\lambda_{p 2}\right)$ & 1563.25 & $\mathrm{~nm}$ \\
Central wavelength $\left(\lambda_{c}\right)$ & 1557.625 & $\mathrm{~nm}$ \\
ZDW $\left(\lambda_{0}\right)$ & 1556.5 & $\mathrm{~nm}$ \\
Second order dispersion coefficient $\beta_{2}$ & $-2.1108 * 10^{-2}$ & $\mathrm{ps}^{2} / \mathrm{km}$ \\
Fourth order dispersion coefficient $\beta_{4}$ & $6.2307 * 10^{-5}$ & $\mathrm{ps}^{4} / \mathrm{km}$ \\
Sixth order dispersion coefficient $\beta_{6}$ & $1.1783 * 10^{-8}$ & $\mathrm{ps}{ }^{6} / \mathrm{km}$ \\
Pump1 power $P_{1}$ & 1 & $\mathrm{~W}$ \\
Pump2 power $P_{2}$ & 0.75 & $\mathrm{~W}$ \\
Power signal $\left(P_{S}\right)$ & -40 & $\mathrm{dBm}$ \\
\hline
\end{tabular}

\section{Results}

Parametric gain spectrum depends on linear phase-mismatch Eq. (8) while Eq. (8) depends on higher-order dispersion coefficient. Also, researchers [19] show an improvement of the bandwidth of FOPA using Photonic Crystal Fiber (PCF) if high order dispersion up to $\beta_{6}$ was considered. Thus, to determine the impact of higher-order dispersion on 2p-FOPA with HNL-DSF, 6 variations of higherorder dispersion were simulated while other parameters follow Table 1 as follows

Case 1: $\beta_{2}$ only (black plot)

Case 2: $\beta_{2}$ and $\beta_{4}$ only (cyan plot)

Case $3 \beta_{2}, \beta_{4}$ and $-\beta_{6}$ (magenta plot)

Case $4:-\beta_{2}$ only (green plot)

Case $5:-\beta_{2}$ and $\beta_{4}$ only (red plot)

Case 6: $-\beta_{2}, \beta_{4}$ and $-\beta_{6}$ (dotted blue plot)

Figure 2 tells that positive $\beta_{2}$ gives poor gain as shown in the black color plot. If positive $\beta_{4}$ (cyan color plot), was added to positive $\beta_{2}$, it worsens the gain that obtained in positive $\beta_{2}$ case. Even negative $\beta_{6}$ (magenta color plot) was added to compensate the positive $\beta_{4}$ and $\beta_{2}$. It gives the same plot as case two due to the small magnitude of $\beta_{6}$ fails to make any changes. On the other hand, if only negative $\beta_{2}$ (greed plot) was considered in linear phase-mismatch as given in the green color plot, it gives better gain than in the first case of positive $\beta_{2}$ inclusion. Further addition of $\beta_{4}$ and negative $\beta_{2}$ terms (red plot) in linear phase-mismatch widen the bandwidth and reduce the ripples if compared to the inclusion of negative $\beta_{2}$ (green plot) term only as reported in [20]. However further inclusion of $\beta_{6}, \beta_{4}$ and negative $\beta_{2}$ (dotted blue plot) gives almost same plot to the case 5 due to the small magnitude of $\beta_{6}$. It is well known that phase matching occurs when $\kappa=\Delta \beta+\gamma\left(P_{1}+P_{2}\right)=0$ which implies $\Delta \beta=-\gamma\left(P_{1}+P_{2}\right)$. As $\gamma\left(P_{1}+P_{2}\right)$ is always positive and Eq. 8 indicates $\beta_{2}$ must be positioned in anomalous region so that $\beta_{2}$ is negative to have a good 
parametric gain. Besides, real parametric gain takes place if $-4 \gamma\left(P_{1}+P_{2}\right)<\Delta \beta<0$ [14]. As portrayed in Figure 3 , when $\beta_{2}$ is positive, $\Delta \beta$ is positive, that is why it gives poor gain as shown in Figure 2 . When $\beta_{2}$ is negative, $\Delta \beta$ is in negative region as shown in Figure 3. Inclusion of $\beta_{4}$ to negative $\beta_{2}$ or case 6 gives wider bandwidth as $\lambda_{s}-\lambda_{c}<-75.62, \beta_{2}$ not in the range of $-4 \gamma\left(P_{1}+P_{2}\right)<\Delta \beta<0$, but inclusion of $\beta_{4}$ to negative $\beta_{2}$ or case 6 still in the range of $-4 \gamma\left(P_{1}+P_{2}\right)<\Delta \beta<0$.

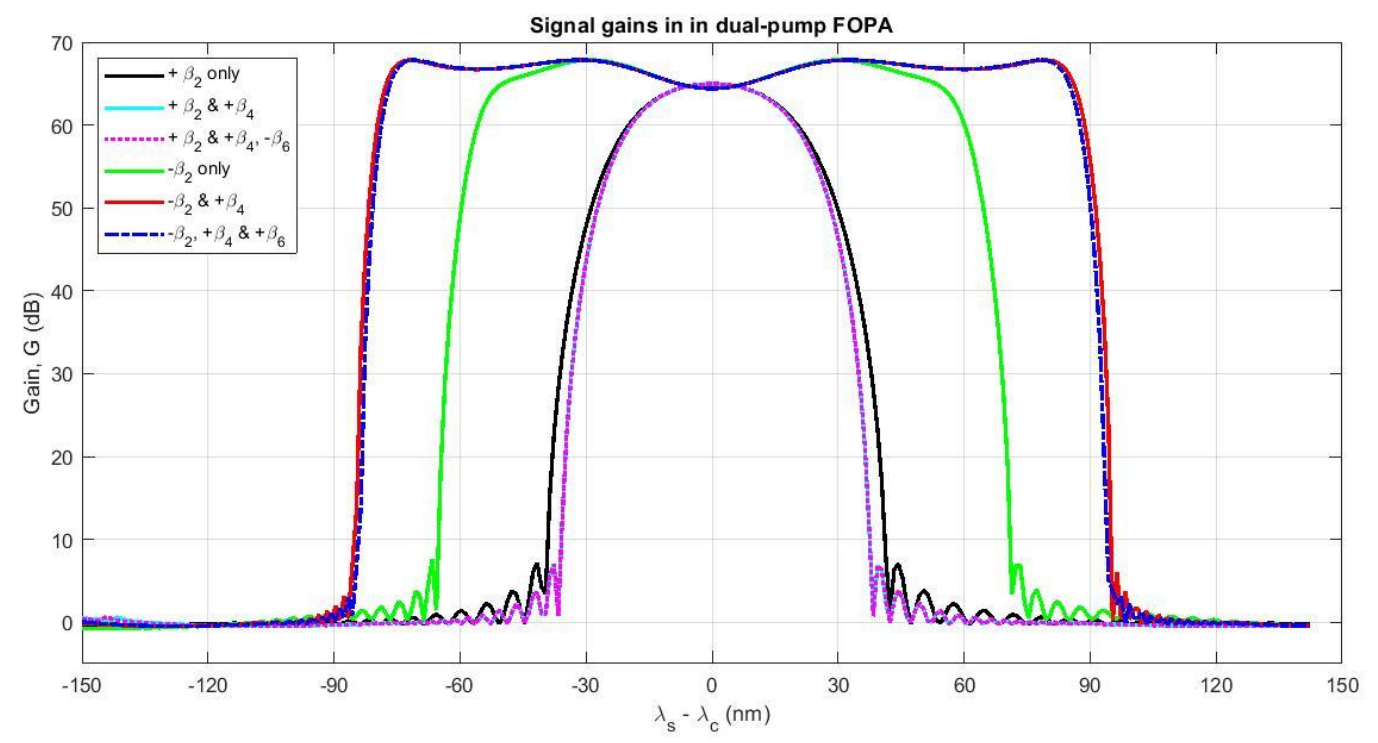

Fig. 2. Effect of higher-order dispersion coefficients

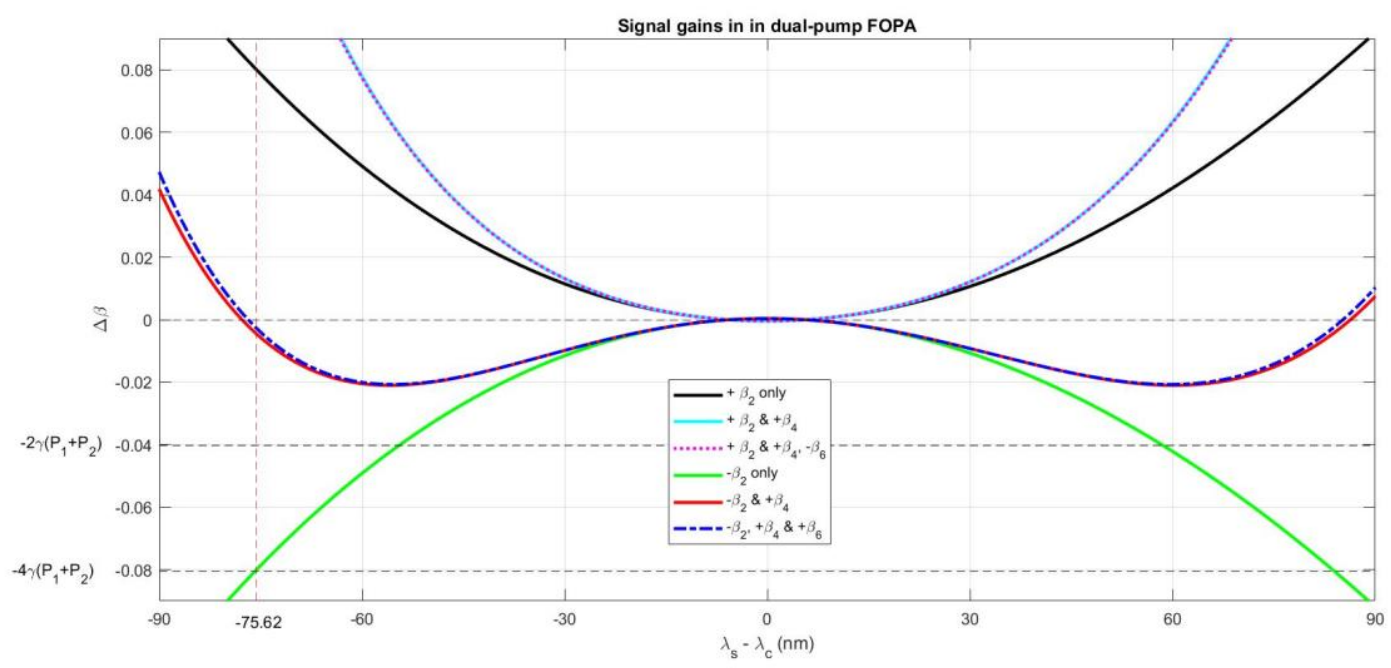

Fig. 3. $\Delta \beta$

In the afford to study effect of fiber length on the gain spectrum, fiber length was varied from $100 \mathrm{~m}$ to $500 \mathrm{~m}$ with and increment of $100 \mathrm{~m}$, while other parameters were fixed as given in Table 1. Figure 4 reveals that by increasing the length of the fiber, the gain will be increased, but the flatness of the gain and bandwidth are reduced. Fiber length $100 \mathrm{~m}$ gives the flatness and widest bandwidth but it has the lowest gain if compared to others. 


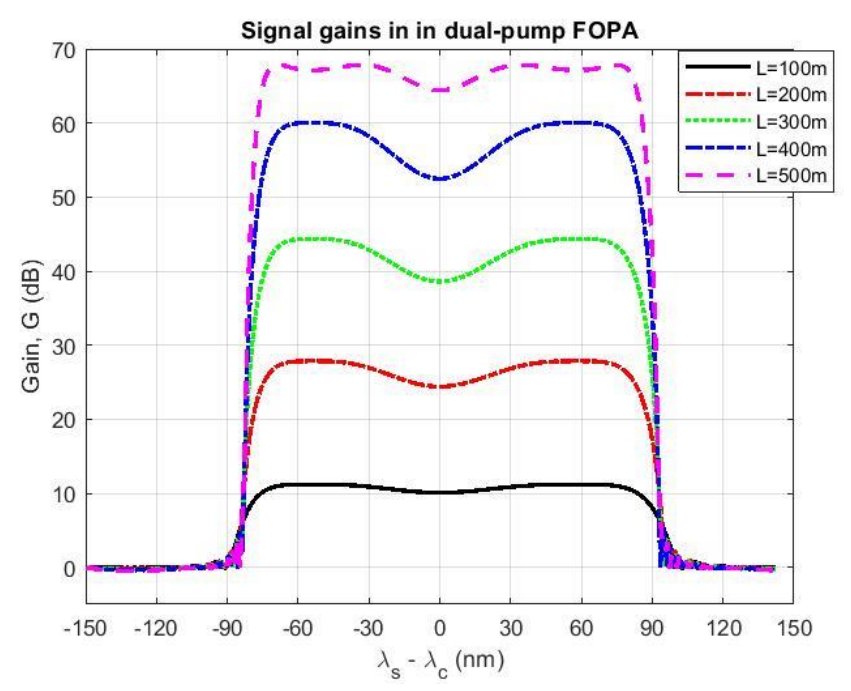

Fig. 4. Effect of fiber length on the gain spectrum

To investigate the impact of the fiber nonlinearity on gain profile, fiber nonlinearity was varied from $7.5 \mathrm{~W}^{-1} \mathrm{~km}^{-1}$ to $11.5 \mathrm{~W}^{-1} \mathrm{~km}^{-1}$ with an increment of $1 \mathrm{~W}^{-1} \mathrm{~km}^{-1}$, while other parameters are the same in Table 1. Figure 5 shows by increasing the values of the nonlinearity of 2 continuous wave pump FOPA, the gain will be increased, flattened and bandwidth is slightly broader. The increase of gain as $\gamma$ increases is evidently from Eq. (3).

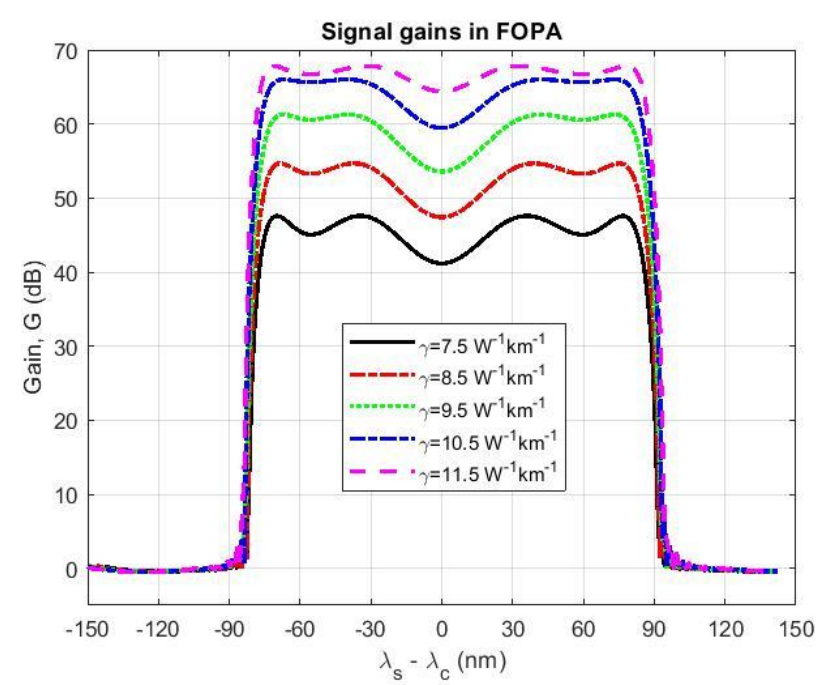

Fig. 5. Effect of fiber nonlinearity

In practice, fiber attenuation exists in fiber optic, thus to study the effect of fiber attenuation on the $2 p$-FOPA gain spectrum, fiber attenuation was varied from $0.82 \mathrm{~dB} / \mathrm{km}, 2 \mathrm{~dB} / \mathrm{km} .3 \mathrm{~dB} / \mathrm{km}$, $4 \mathrm{~dB} / \mathrm{km}$ and $5 \mathrm{~dB} / \mathrm{km}$ as displayed in Figure 6 while other parameters were fixed as given in Table 1. The gain damps as fiber attenuation is increased. It is due to if only the last term in RHS of Eq. 3 is focused, an increase of fiber attenuation $\alpha$ will give a decay of the amplitude of the signal because of the negative sign of Eq. (9) and thus gain as gain $G$ depends on $A_{s}$.

$$
\frac{d A_{s}}{d z}=-\frac{\alpha}{2} A_{s}
$$




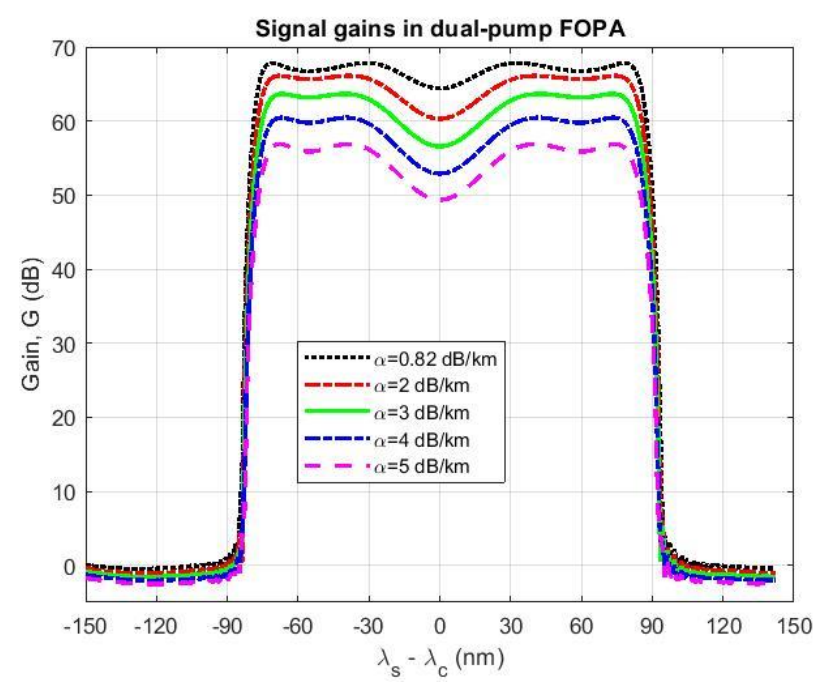

Fig. 6. Effect of fiber loss

Figure 7 gives the influence of pump power by fixing pump power $P_{1}$ to $1 \mathrm{~W}$, while varying pump power $P_{2}$ from $0.2 \mathrm{~W}$ to $0.8 \mathrm{~W}$ with an increment of $0.2 \mathrm{~W}$. The other parameters follow Table 1 . It tells that increase of power will increase gain, flatten gain at wavelength far from the central wavelength and reduce the gain drops at a wavelength near the central wavelength. It is well known that maximum gain occurs if total phase mismatch $\kappa=0$. Figure 8 shows $\kappa$ for the case of $P_{1}=1 \mathrm{~W}$ and $P_{2}=0.8 \mathrm{~W}$ is the closest to zero if compared to the other three cases, so its gain drops at its wavelength near the central wavelength drops is the minimum.

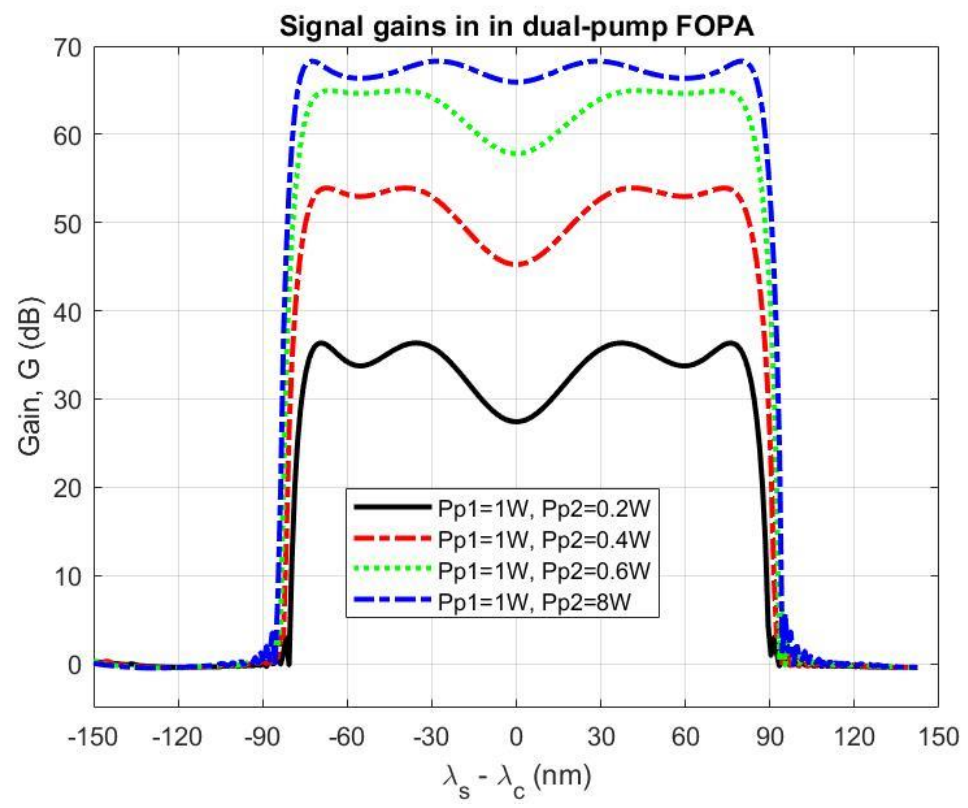

Fig. 7. Effect of pump powers 


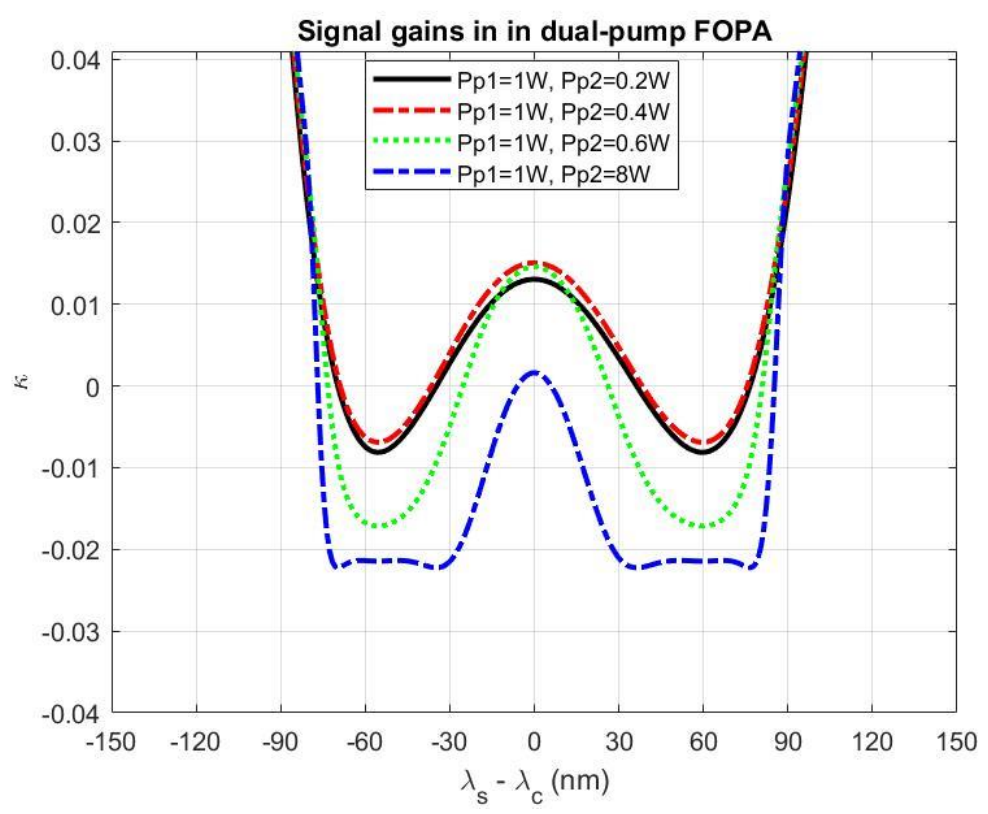

Fig. 8. Effect of pump powers

It is crucial to determine pump wavelength that would represent the minimal pump wavelength separation $\Delta \lambda_{p}=\lambda_{p 2}-\lambda_{p 1}$ to the ZDW of the HNLF to ensure that high, flat and broad gain as possible. It is because if the pumps are placed far from the ZDW, lower gain will be obtained [2]. To optimize $\Delta \lambda_{p}, \Delta \lambda_{p}$ was varied from $11.25 \mathrm{~nm}$ to $41.25 \mathrm{~nm}$ with an increment of $10 \mathrm{~nm}$, while $\lambda_{c}$ and other parameters were fixed as given in Table 1 . Figure 9 tells as $\Delta \lambda_{p}$ increases, the reduction of gain at the central wavelength is significantly increased too, but gain at wavelength far from the central wavelength is flattened similar to [14].

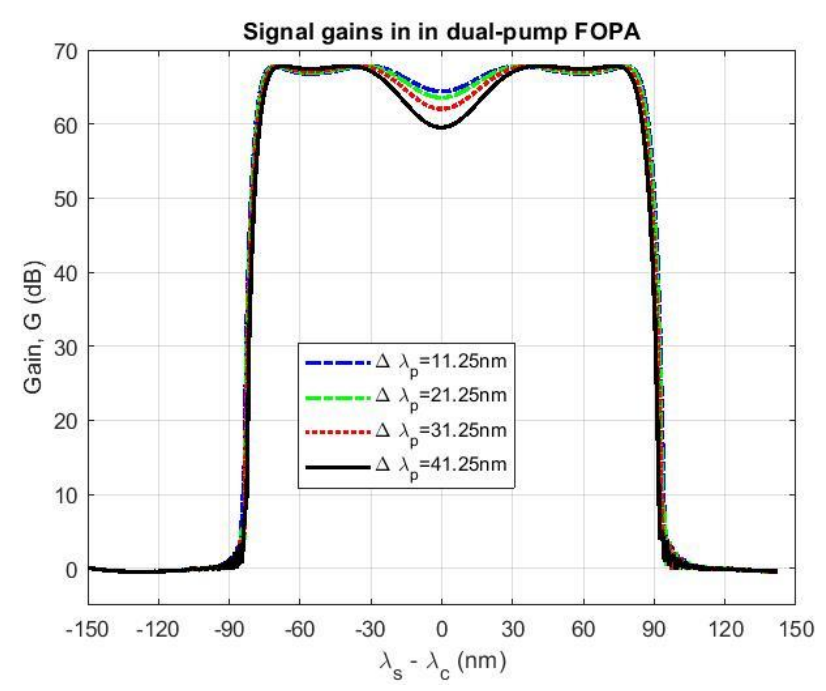

Fig. 9. Effect of $\Delta \lambda_{p}$

The placement of pump wavelength will determine the central wavelength $\lambda_{c}$ and hence $\lambda_{c}-\lambda_{0}$ which has an impact on gain performance [21]. For this purpose, by maintaining $\Delta \lambda_{p}=11.25 \mathrm{~nm}$ and other parameters as given in Table 1 while varying $\lambda_{p 1}$ and $\lambda_{p 2}$, a total 8 cases of $\lambda_{c}-\lambda_{0}$ were investigated as seen in Figure 10. Table 2 gives values of $\beta_{2}, \beta_{4}, \beta_{6}$ for each case of $\lambda_{c}-\lambda_{0}$. Figure 9 
shows when $\lambda_{c}-\lambda_{0}$ is negative or zero, there is poor gain as positive $\beta_{2}$ value shows it is in a normal dispersion region which yields poor gain if compared to in the anomalous dispersion region (negative $\beta_{2}$ value). An increase of $\lambda_{c}-\lambda_{0}$ from $0.5 \mathrm{~nm}$ to $2 \mathrm{~nm}$ yields an increase of bandwidth of gain, however there is a significant reduction of gain at wavelength far from central wavelength for the cases of $\lambda_{c}-\lambda_{0}=1.5 \mathrm{~nm}, 1.625 \mathrm{~nm}$ and $2 \mathrm{~nm}$. The case $\lambda_{c}-\lambda_{0}=2 \mathrm{~nm}$ shows the highest reduction of gain at wavelength far from central wavelength until it forms both-side narrow gain.

Table 2

Values of $\beta_{2}, \beta_{4}, \beta_{6}$

\begin{tabular}{llll}
\hline$\lambda_{c}-\lambda_{0}(\mathrm{~nm})$ & $\beta_{2}\left(s^{2} / \mathrm{m}\right)$ & $\beta_{4}\left(\mathrm{~s}^{4} / \mathrm{m}\right)$ & $\beta_{6}\left(\mathrm{~s}^{6} / \mathrm{m}\right)$ \\
\hline-1.125 & $2.2169 \times 10^{-29}$ & $6.0182 \times 10^{-56}$ & $1.1602 \times 10^{-83}$ \\
0 & $4.9139 \times 10^{-29}$ & $6.1241 \times 10^{-56}$ & $1.1692 \times 10^{-83}$ \\
0.5 & $-9.1182 \times 10^{-29}$ & $6.1714 \times 10^{-56}$ & $1.1733 \times 10^{-83}$ \\
1 & $-1.8712 \times 10^{-29}$ & $6.2188 \times 10^{-56}$ & $1.1773 \times 10^{-83}$ \\
1.125 & $-2.1108 \times 10^{-29}$ & $6.2307 \times 10^{-56}$ & $1.1783 \times 10^{-83}$ \\
1.5 & $-2.8291 \times 10^{-29}$ & $6.2664 \times 10^{-56}$ & $1.1814 \times 10^{-83}$ \\
1.625 & $-3.0883 \times 10^{-29}$ & $6.2783 \times 10^{-56}$ & $1.1824 \times 10^{-83}$ \\
2 & $-3.7854 \times 10^{-29}$ & $6.3141 \times 10^{-56}$ & $1.1854 \times 10^{-83}$ \\
\hline
\end{tabular}

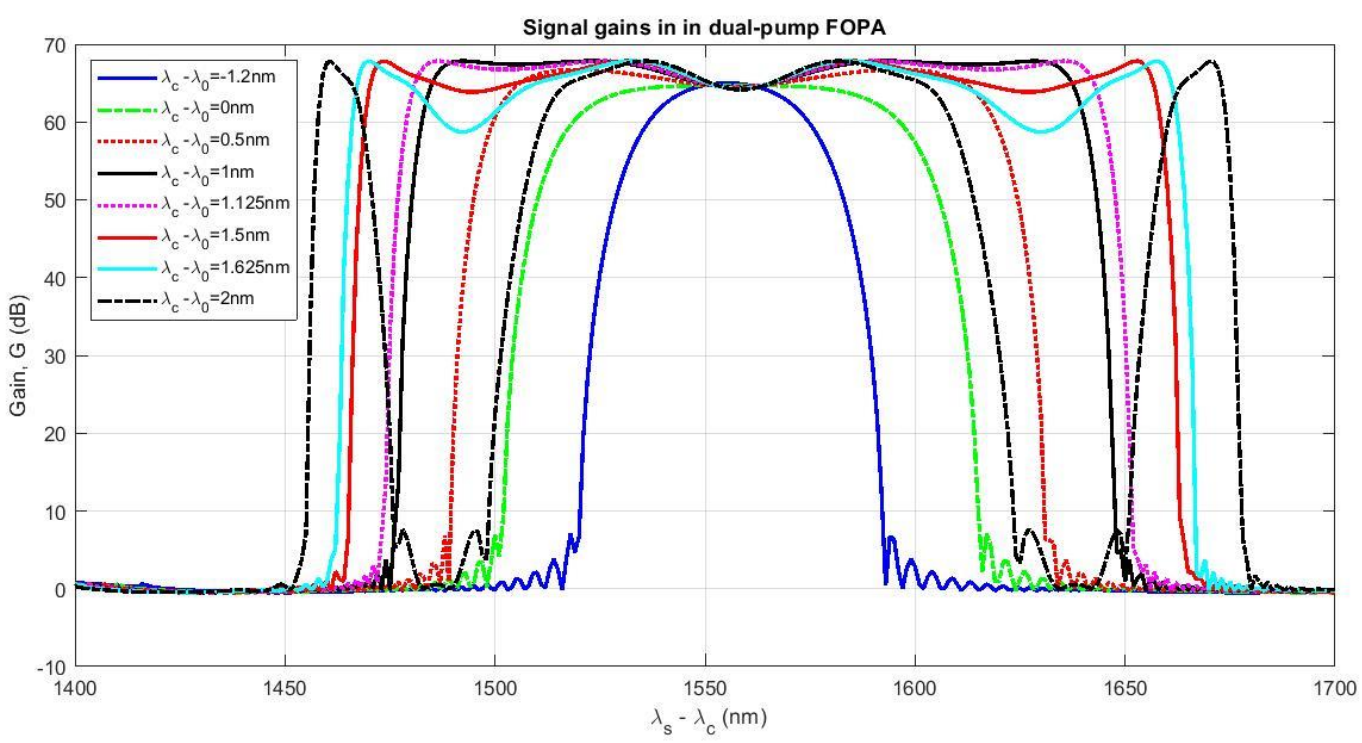

Fig. 10. Effect of $\lambda_{c}-\lambda_{0}$

\section{Conclusions}

Seven parameters (higher-order dispersion coefficients, fiber length, fiber nonlinearity, fiber attenuation, pump powers, pump wavelength separation $\Delta \lambda_{p}$ and distance of central pump wavelength with ZDW $\lambda_{c}-\lambda_{0}$ ) which have influenced the gain (9) performance of $2 p$ - FOPA have been numerically investigated by solving the four coupled amplitude equations (1-4) which governs the process of FWM with linear phase mismatch (8) using ode45 function of Matlab 2019b. It is found that positive $\beta_{2}$ gives poor or no gain. Meanwhile, the addition of positive $\beta_{4}$ to positive $\beta_{2}$ worsens the gain. Even negative $\beta_{6}$ was added to both positive $\beta_{4}$ and $\beta_{2}$, it still gives the same gain as in the addition of positive $\beta_{4}$ and positive $\beta_{2}$ only. This may due to the small magnitude of $\beta_{6}$ doesn't 
make any changes. The addition of $\beta_{4}$ to negative $\beta_{2}$ widens the bandwidth, but there is no significant effect with the addition of $\beta_{6}$. An increment of fiber length, fiber nonlinearity and pump powers give a significant gain improvement, but when fiber attenuation is increased, the gain amplitude drops. An increase of pump separation improves signal gain at wavelength far from the central wavelength but give reduction at the central wavelength. Lastly, $\lambda_{c}-\lambda_{0}$ must be positive and not $>1.125 \mathrm{~nm}$ to get a broader gain and not gain reduction at wavelength far from the central wavelength.

\section{Acknowledgement}

The authors would like to thank the Ministry of Higher Education Malaysia for supporting this research under the fundamental research grant scheme FRGS/1/2018/TK04/UTHM/02/13 and partially sponsored by Universiti Tun Hussein Onn Malaysia.

\section{References}

[1] Marconi, J. D., Boggio, J. C., Fragnito, H. L., \& Bickham, S. R. (2007, March). Nearly 100 nm bandwidth of flat gain with a double-pumped fiber optic parametric amplifier. In OFC/NFOEC 2007-2007 Conference on Optical Fiber Communication and the National Fiber Optic Engineers Conference (pp. 1-3). IEEE. https://doi.org/10.1109/OFC.2007.4348839

[2] Olonkins, S., Bickovs, V., Salgals, T., Bobrovs, V. and Ivanovs, G. "Investigation of Dual-Pump FOPA Performancein a 4-Channel 40 Gbps WDM Transmission System." Elektronika Ir Elektrotechnika 23 (2017): 85-89. https://doi.org/10.5755/i01.eie.23.6.19699

[3] Marhic, Michel E., Peter A. Andrekson, Periklis Petropoulos, Stojan Radic, Christophe Peucheret, and Mahmoud Jazayerifar. "Fiber optical parametric amplifiers in optical communication systems." Laser \& photonics reviews 9 , no. 1 (2015): 50-74. https://doi.org/10.1002//por.201400087

[4] Saris, Nur Najahatul Huda, Azura Hamzah, and Sumiaty Ambran. "Investigation on Gain Improvement of Erbium Doped Fiber Amplifier (EDFA) By Using Dual Pumped Double Pass Scheme." Journal of Advanced Research in Applied Sciences and Engineering Technology 7, no. 1 (2017): 11-18.

[5] Wong, Ngai, and Kenneth K-Y. Wong. "Gain bandwidth optimization in two-pump fiber optical parametric amplifiers under bounded zero-dispersion wavelength fluctuations." Optics communications 272, no. 2 (2007): 514-520. https://doi.org/10.1016/i.optcom.2006.11.057

[6] Zhu, Z., Zhu, H. Sui, H., Cheng, L., Yu, L., Zhang, Y., Taccheo, S., and Luo, B. "Influence of chirped pump shape on the two-pump fiber optical parametric amplification." Journal of the Optical Society of America B 37 (2020): 2464-2470. https://doi.org/10.1364/JOSAB.399524

[7] Zuan, A. M. S., M. K. Z. Anuar, S. Syahrullail, M. N. Musa, and E. A. Rahim. "A study of float wave energy converter (FWEC) model." Journal of Advanced Research in Applied Sciences and Engineering Technology 1, no. 1 (2015): 4049.

[8] Maji, Partha Sona, and Partha Roy Chaudhuri. "Gain and bandwidth investigation in a near-zero ultra-flat dispersion PCF for optical parametric amplification around the communication wavelength." Applied optics 54, no. 11 (2015): 3263-3272. https://doi.org/10.1364/A0.54.003263

[9] Chen, H. Y., Zhang, Z. X., Sang M. H. and Zhan, L. "Gain Characteristic of a Dual-Pump Fiber-Optical Parametric Amplifier with a Highly Nonlinear Fiber." Laser Physics 18 (2008): 983-987. https://doi.org/10.1134/S1054660X08080124

[10] Kaur, Gaganpreet, Gurmeet Kaur, and Sanjay Sharma. "Performance Investigation of Dual pump Fiber Optical Parametric amplifier for Flat gain over $220 \mathrm{~nm}$ Gain Bandwidth." An International Journal of Engineering Sciences 17 (2016): 451-457.

[11] Chavez. Boggio, J. M., Marconi, J. D. and Fragnito, H. L. "Double-Pumped Fiber Optical Parametric Amplifier with Flat Gain Over 47-nm Bandwidth Using a Conventional Dispersion-Shifted Fiber." IEEE Photonics Technology Letters 17 (2005): 1842-1844. https://doi.org/10.1109/LPT.2005.852334

[12] Kipnoo, E. K. R., Waswa, D., Amolo, G. and Leitch, A.W. R. "Gain Analysis for a 2-Pump Fibre Optical Parametric Amplifier." The African Review of Physics 9 (2014): 47-52.

[13] Myint, S., Lwin, Z. M and Tun, H. M. "Performance Analysis of Single-Pumped and Dual-Pumped Parametric Optical Amplifier." International Journal of Scientific \& Technology Research 4 (2015): 381-386. 
[14] Othman, N., NS Mohd Shah, K. G. Tay, N. A. Cholan, and R. Talib. "The effect of pump parameters on dual-pump fiber optical parametric amplifier." In MATEC Web of Conferences, vol. 150, p. 01010. EDP Sciences, 2018. https://doi.org/10.1051/matecconf/201815001010

[15] Marhic, M. E_, Y. Park, F. S. Yang, and L. G. Kazovsky. "Broadband fiber-optical parametric amplifiers and wavelength converters with low-ripple Chebyshev gain spectra." Optics letters 21, no. 17 (1996): 1354-1356. https://doi.org/10.1364/OL.21.001354

[16] Chatterjee, Sudip K., Saba N. Khan, and Partha Roy Chaudhuri. "Two-octave spanning single pump parametric amplification at $1550 \mathrm{~nm}$ in a host lead-silicate binary multi-clad microstructure fiber: influence of multi-order dispersion $\quad$ engineering." Optics $\quad$ Communications $332 \quad$ (2014): https://doi.org/10.1016/i.optcom.2014.07.021

[17] Tay, K. G., Othman, N., Shah, N.S.M. and Cholan, N.A. "Numerical Simulation of Highly-Nonlinear Dispersion-Shifted Fiber Optical Parametric Gain Spectrum with Fiber Loss and Higher-Order Dispersion Coefficients." Telkomnika 15 (2017): 1461 - 1469. https://doi.org/10.12928/telkomnika.v15i3.7215

[18] Hayytov, Serdar, Wah Yen Tey, Hooi Siang Kang, Mohammed W. Muhieldeen, and Omid Afshar. "Comparative Review on Computational Performance of Multistep Schemes in Solving One-Dimensional Linear Wave Equation." CFD Letters 13, no. 6 (2021): 1-14. https://doi.org/10.37934/cfdl.13.6.114

[19] Dainese, P., Wiederhecker, G. S., Rieznik, A. A., Fragnito, H.L, Hernández-Figueroa, H. E. “Designing fiber dispersion for broadband parametric amplifiers." SBMO/IEEEMTT-S International Microwave and Optoelectronics Conference Proceedings 200592 (2005):1-4.

[20] Vedadi, A., Marhic, M. E., Lantz, E., Maillotte, H., and Sylvestre, T. "Investigation of gain ripple in two-pump fiber optical parametric amplifiers." Optics Letters 33, no. 19, (2008): 2203-2205. https://doi.org/10.1364/OL.33.002203

[21] Parolari, Paola, Lucia Marazzi, Elisabetta Rognoni, and Mario Martinelli. "Influence of pump parameters on twopump optical parametric amplification." Journal of lightwave technology 23, no. 8 (2005): 2524. https://doi.org/10.1109/JLT.2005.850018 